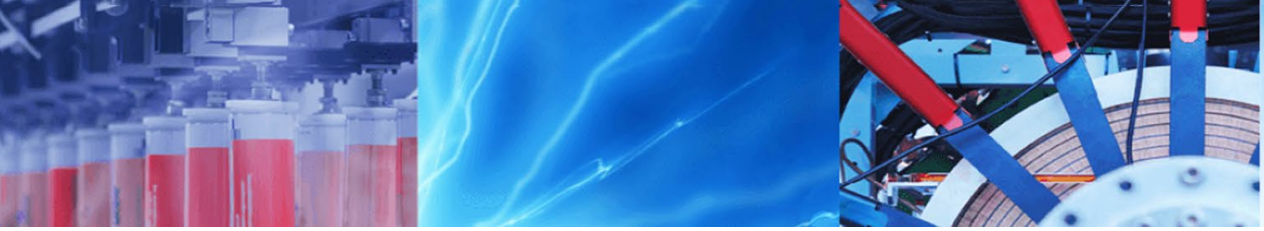

Research Article

\title{
Fabrication and performance of an ultrafine silver grid film applied to flexible touch sensor
}

\author{
Genchu Tang ${ }^{1,2}$ (D) $\cdot$ Bin Tang ${ }^{1,2}$
}

Received: 23 October 2019 / Accepted: 26 November 2019 / Published online: 17 December 2019

(C) The Author(s) 2019 OPEN

\begin{abstract}
The fabrication and performance of an ultrafine silver (Ag) grid film applied to flexible touch sensor as the transparent conductive electrode is reported. The ultrafine Ag grid film was fabricated based on the laser direct writing, electroforming, nano-imprint lithography. In the manufacturing process, firstly, the Nickel ( $\mathrm{Ni}$ ) mold used as the master mold was obtained by laser direct writing and electroforming technologies. Secondly, the micro-grooves were transferred from the Ni mold onto the surface of UV glue coated on the polyethylene terephthalate film through nano-imprinting technology. Lastly, the ultrafine Ag grid was generated through nano-imprint lithography with Ag paste filled into the micro-grooves on the UV glue. The result indicated that the ultrafine Ag grid film with size (L) $640 \mathrm{~mm} \times$ (W) $520 \mathrm{~mm}$ had a uniform line width of $1.01 \mu \mathrm{m}$ and showed excellent optoelectronic and mechanical properties, such as optical transmittance $90.00 \%$, Haze $1.49 \%$, sheet resistance $5.4 \Omega / \square$, the variation ratio of the sheet resistance within $3 \%$ after 8000 bending cycles, and the almost negligible morphology change after the adhesion cross-cut test. Furthermore, the functional test was performed on a flexible touch sensor applying the ultrafine Ag grid film.
\end{abstract}

Keywords Ultrafine silver grid film · Flexible touch sensor · Transparent conductive electrode · Nickel mold · Laser direct writing

\section{Introduction}

As a pivotal component of many photoelectric products described by Chung et al. [1] including solar cells studied by Yang et al. [2], liquid crystal displays (LCDs) reported by Blake et al. [3], organic light-emitting diodes(OLEDs) shown by Wu et al. [4] and touch screen panels (TSPs) discussed by Jeon et al. [5] and Kim et al. [6], conductive electrodes have attracted more and more attention. Indium tin oxide (ITO) is the most widely used transparent conductive electrode material owing to its excellent optoelectronic properties. However, as Hecht et al. [7] and Kwon et al. [8] proposed, many new applications have been limited due to its disadvantages, such as its brittleness and scarcity. Because of the flexible, abundant and cost-effective, transparent conductive electrode material will undoubtedly become an irresistible trend, which researched by Tang et al. [9], Li et al. [10] and Lai et al. [11].

Thus, the next generation of material with superior flexibility and abundant resources, without damaging the optoelectronic properties of transparent conductive electrode materials, is urgently needed. Current researches on alternative materials mainly focusing on carbon nanotubes reported by Yu et al. [12], graphene described by Bae et al. [13] and Tung et al. [14], conducting polymers investigated by Kirchmeyer and Reuter [15], polymer composite to improve the long-term stability of the conductive film was researched by Jiang et al. [16, 17], metal nanostructures shown by Gaynor et al. [18], Hong et al. [19] and Madaria et al. [20]. While the conductivity and

Genchu Tang, tanggenchu@ofilm.com | 'Postdoctoral Programme of O-Film Technology Co., Ltd., Nanchang 330032, People's Republic of China. ${ }^{2}$ O-Film Technology Co., Ltd., Nanchang 330032, People's Republic of China. 
transmittance of carbon nano-materials are poorer than those of metal nanostructures, the same with the conductivity and stability of conducting polymers based on the researches of Hecht et al. [7] and Kumar and Zhou [21]. And metal nanostructures, such as Ag nanowires or grids, have been successfully applied in touch screen panel based on the reports of Madaria et al. [20], Hong et al. [22] and Hyunjin et al. [23]. However, there are still shortcomings in a few crucial properties of the Ag nanostructures in their reports, such as the line width of Ag nanowires, optical transmittance and haze, sheet resistance, mechanical stability and so on. Therefore, the further improvement of these properties will be critical to the effective application of Ag nanostructures in the practical industrial manufacturing.

In the present work, the fabrication and performance of an ultrafine silver $(\mathrm{Ag})$ grid film applied to flexible touch sensor as the transparent conductive electrode was introduced. In order to improve the deficiencies of the above mentioned properties, and realize the application of Ag nanostructures in the large-area, high-throughput and low-cost production of flexible touch sensor. The ultrafine Ag grid film was fabricated based on the laser direct writing, electroforming, nano-imprint lithography. In the manufacturing process, the Nickel (Ni) mold used as the master mold was obtained by laser direct writing and electroforming technologies, and the micro-grooves were transferred from the $\mathrm{Ni}$ mold onto the surface of UV glue coated on the polyethylene terephthalate (PET) film through the nano-imprint lithography. Moreover, the ultrafine Ag grid was generated through nano-imprint lithography with $\mathrm{Ag}$ paste filled into the micro-grooves on the UV glue and baked at $130^{\circ} \mathrm{C}$ for $1 \mathrm{~h}$. In addition, the properties of the fabricated ultrafine Ag grid film were well studied and performed in order to evaluate the possibility of the fabricated ultrafine Ag grid film substituting for the conventional ITO electrode, and being adopted as a promising transparent conductive electrode applied to the flexible touch sensor.

\section{Experimental}

\subsection{Fabrication of Ni mold}

The Ni mold played an important role in the nano-imprinting process, especially the performance of the ultrafine Ag grid film was determined by the accuracy of the pattern on mold. In this work, the fabricated process of the $\mathrm{Ni}$ mold can be divided into the following five steps, and the experimental parameters corresponding to each step are the optimal values obtained from a series of optimization experiments before the actual mass production.
Firstly, the positive photoresist (PR, xingtek, China, $0.7 \mu \mathrm{m}$ ) was coated in $1 \mu \mathrm{m}$ thickness onto the glass substrate $(\mathrm{L})$ $670 \mathrm{~mm} \times(\mathrm{W}) 550 \mathrm{~mm} \times(\mathrm{T}) 4.8 \mathrm{~mm}$ ) by spin-coating, then baked at $100^{\circ} \mathrm{C}$ for $60 \mathrm{~s}$ (Fig. 1a). Secondly, the laser direct writing scanning was performed with the positive PR side of the sample exposed to Laser, the energy density and laser exposure accuracy of the corresponding laser direct writer (DWL, Heidelberg Instruments, German) are $140 \mathrm{~mJ} /$ $\mathrm{cm}^{2}$ and $700 \mathrm{~nm}$, respectively (Fig. 1b). Thirdly, the sample was developed in the developer solution for $50 \mathrm{~s}$ at $25^{\circ} \mathrm{C}$ (Fig. 1C), then baked for $0.5 \mathrm{~h}$ at $120^{\circ} \mathrm{C}$. Fourthly, as shown in Fig. 1d, a thin layer of Ni film was vapor deposited onto the pattern on the positive PR as the cathode of the subsequent electroforming process, and the schematic diagram of the vacuum evaporation device is shown in the top right corner of Fig. 1d. The vacuum evaporation refers to a layer of $\mathrm{Ni}$ with a thickness of about 20-50 nm deposited onto the pattern on the positive photoresist. The highly purified $\mathrm{Ni}$ target materials and extremely cleanness of the vacuum evaporation device are important guarantee for the quality of evaporation. Finally, as shown in Fig. 1e, the Ni mold was generated on the cathode of the electrolytic cell through electroforming technology, meanwhile the schematic diagrams of the electroformed product (the upper part is the corresponding profile of the sample) and electroforming device are shown in the lower left corner and upper right corner of Fig. 1e, respectively. The electroforming process means that the positive photoresist with the surface of evaporated $\mathrm{Ni}$ is placed in the electrolytic cell, a layer of $\mathrm{Ni}$ with a thickness of about $0.2 \mathrm{~mm}$ is electroformed on the positive photoresist, and the positive photoresist is peeled off to obtain the Ni mold. The size of the generated Ni mold was (L) $640 \mathrm{~mm} \times(\mathrm{W})$ $520 \mathrm{~mm} \times(\mathrm{T}) 200 \mu \mathrm{m}$.

\subsection{Fabrication of ultrafine Ag grid film}

The following fabricated process of the ultrafine Ag grid film in this work can be divided into the following three steps.

Firstly, a UV curable synthetic resin (UV glue) prepolymer was coated on the surface of obtained Ni mold, and a PET film with size (L) $640 \mathrm{~mm} \times$ (W) $520 \mathrm{~mm} \times(\mathrm{T})$ $23 \mu \mathrm{m}$ was imprinted with the UV glue by roller. In this process, the coating thickness of the UV glue was directly determined by the viscosity of UV glue, the pressure and rotation speed of the roller. In order to accurately obtain the expected UV glue thickness, a series of inquiry experiments on the relationship between these three impact factors and the coating thickness of the UV glue had been conducted, meanwhile the corresponding relationship model between them was established. 
Fig. 1 The fabrication process of Ni mold in this work: a Positive PR coated onto the glass substrate by spin coating; $\mathbf{b}$ Exposure by Laser; c Developing process; d Vacuum evaporation process; e Electroforming process
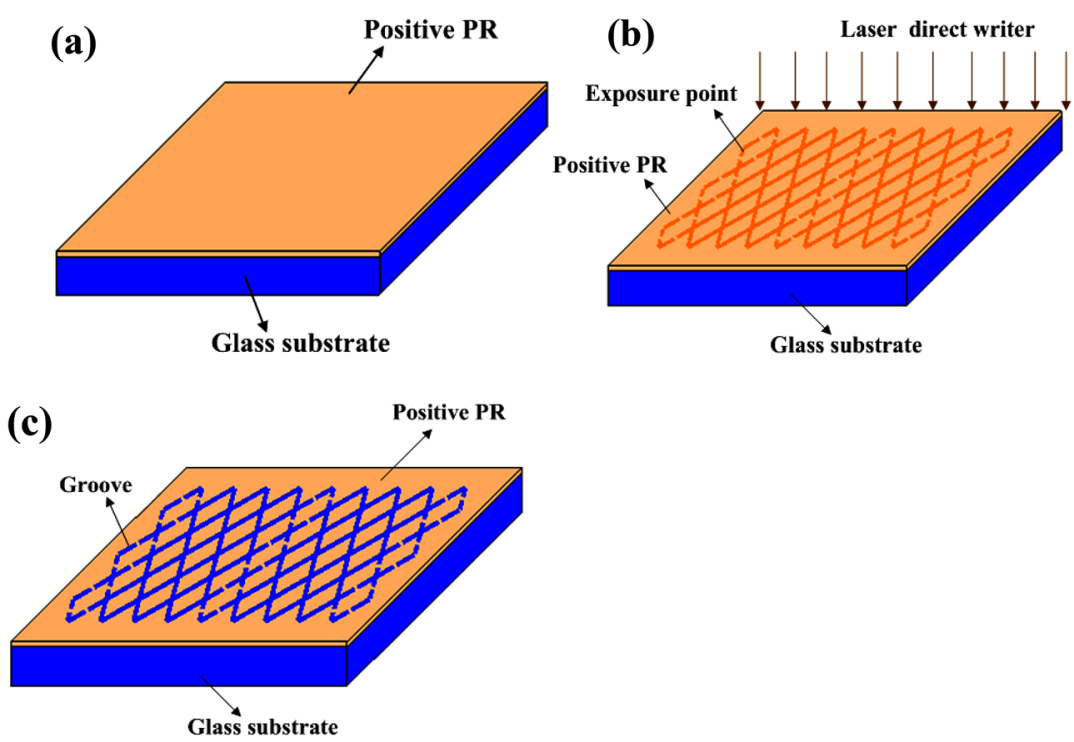

(d)
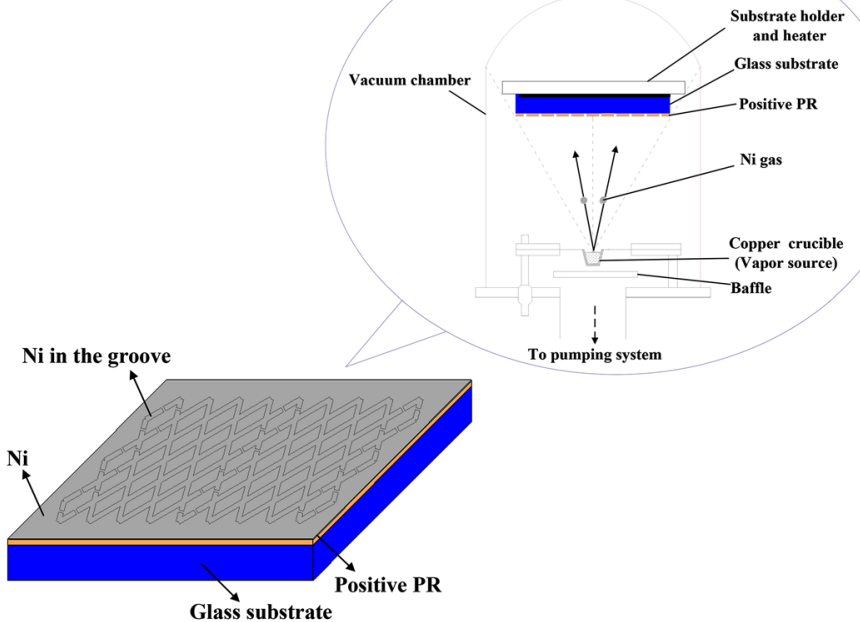

(e)
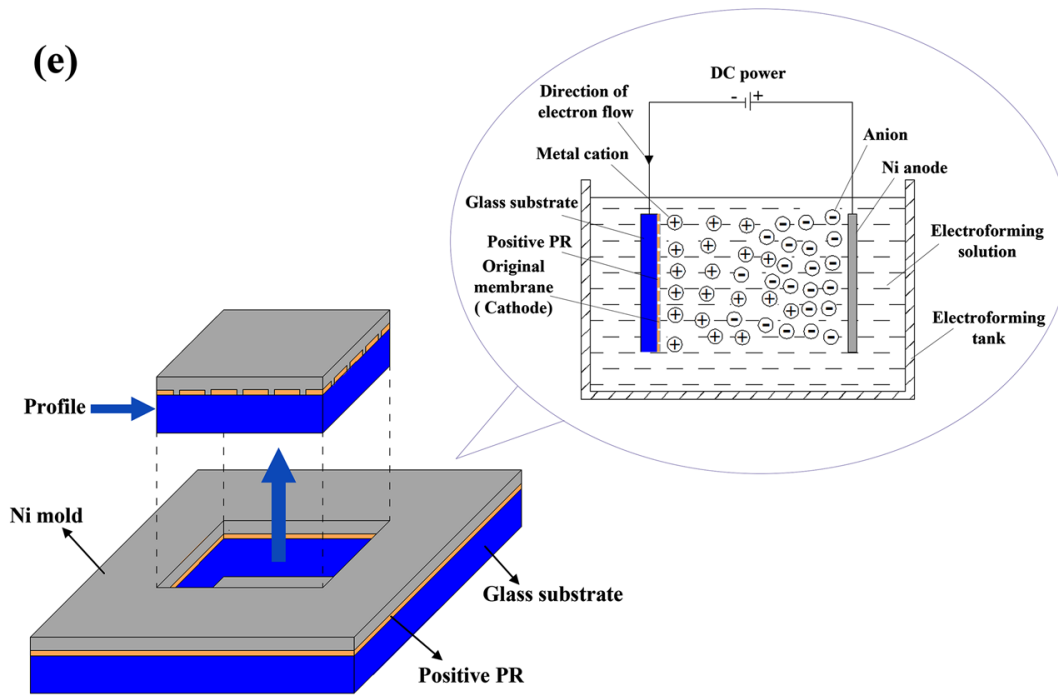
Secondly, the UV glue was cured by exposure to the UV light with an energy density of $600 \mathrm{~mJ} / \mathrm{cm}^{2}$ as shown in Fig. 2a (the upper part is the corresponding profile of the sample), then the micro-grooves were perfectly transferred from the Ni mold onto the UV glue coated on the PET film after the peeling between the PET with UV glue and $\mathrm{Ni}$ mold as shown in Fig. 2b. In this process, the peeling effect was directly determined by the energy density of the UV light, an inappropriate UV light energy density would lead to not only the poor peeling effect, resulting in a poor product, but also the block of the micro-grooves on the Ni mold caused by the residual UV glue, resulting in damaging the Ni mold. In addition, after the peeling process, the surface of the cured UV glue was re-exposed by the UV light with much higher energy density to make sure the product with excellent function and appearance can be obtained in the subsequent printing process.

Lastly, the ultrafine Ag grid film was obtained with the Ag pastes filled into the micro-grooves on the UV glue (Fig. 2c) and baked at $130^{\circ} \mathrm{C}$ for $1 \mathrm{~h}$. In this process, the function and appearance of the product are determined by the pressure and speed of the squeegee used for printing, the leveling of the printing table, the time interval and the wiping force after the printing, the time and temperature of the post-bake, thus the experimental parameters corresponding to each step are the optimal values obtained from a series of optimization experiments before the actual mass production.

\section{Results and discussion}

\subsection{Characterization of the micro-grooves}

As the basis of the ultrafine Ag grid, the micro-grooves play an important role throughout the manufacturing process. Moreover the detailed description of the microgroove is shown as follows. Figure 3 shows the images of the micro-grooves on the UV glue coated on the PET film. As shown in Fig. 3a, there are 12 units on the PET film, and the units with special pattern can be designed for various touch sensors. Moreover, the micro-grooves in the patterns are of the same specification. Figure $3 b, c$ are the micrographs of micro-grooves in different enlarged scales observed with the scanning electron microscope (SEM, S-4800, Hitachi, Japan), it could be found that the microgrooves are uniform, and the pitch and angle is $181.0 \mu \mathrm{m}$ and $19.9^{\circ}$, respectively. In this work, the sheet resistance is $1.95 \Omega / \square$ at the angle $19.9^{\circ}$, which is much lower than most of the reported sheet resistances of transparent conductors used in flexible applications. In the comparative experiments, the sheet resistance is $9.3 \Omega / \square$ at the angle $60^{\circ}$, the sheet resistance is $5.4 \Omega / \square$ at the angle $45^{\circ}$, and the sheet resistance is $3.1 \Omega / \square$ at the angle $30^{\circ}$. Furthermore, the micro-grooves in the rhombus pattern at the angle $19.9^{\circ}$ can improve the pixel resolution, and also solve the interference effect of moire fringes. Figure $3 \mathrm{~d}$ illustrates the measurement of the width $(1.01 \mu \mathrm{m})$ and depth $(1.02 \mu \mathrm{m})$ of a micro-groove on the UV glue observed with the shape-measuring laser microscope (SLM, VK-X 210, Keyence, Japan).
Fig. 2 The fabrication process of the ultrafine Ag grid film in this work: a Coating and curing process of UV glue; $\mathbf{b}$ Peeling between the PET with UV glue and Ni mold; c Filling process of Ag paste
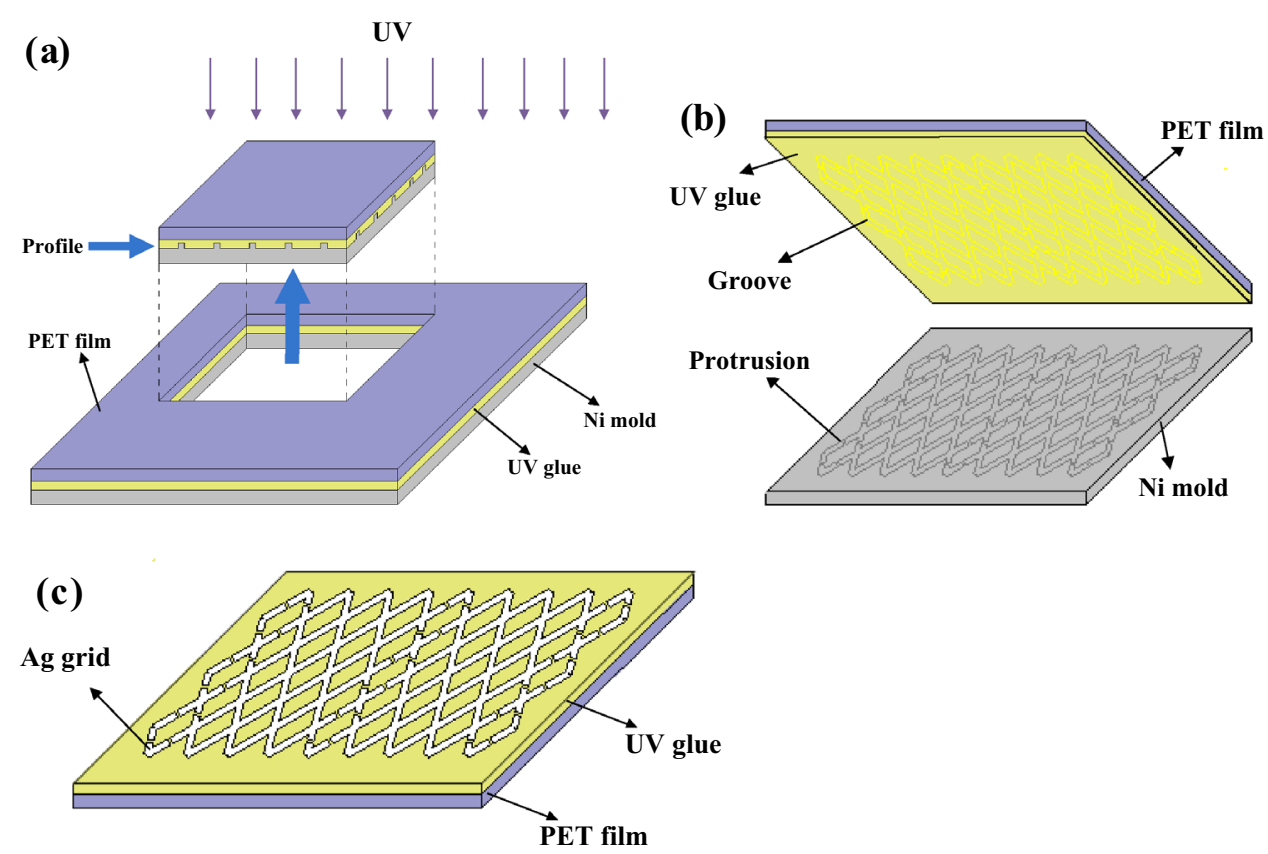

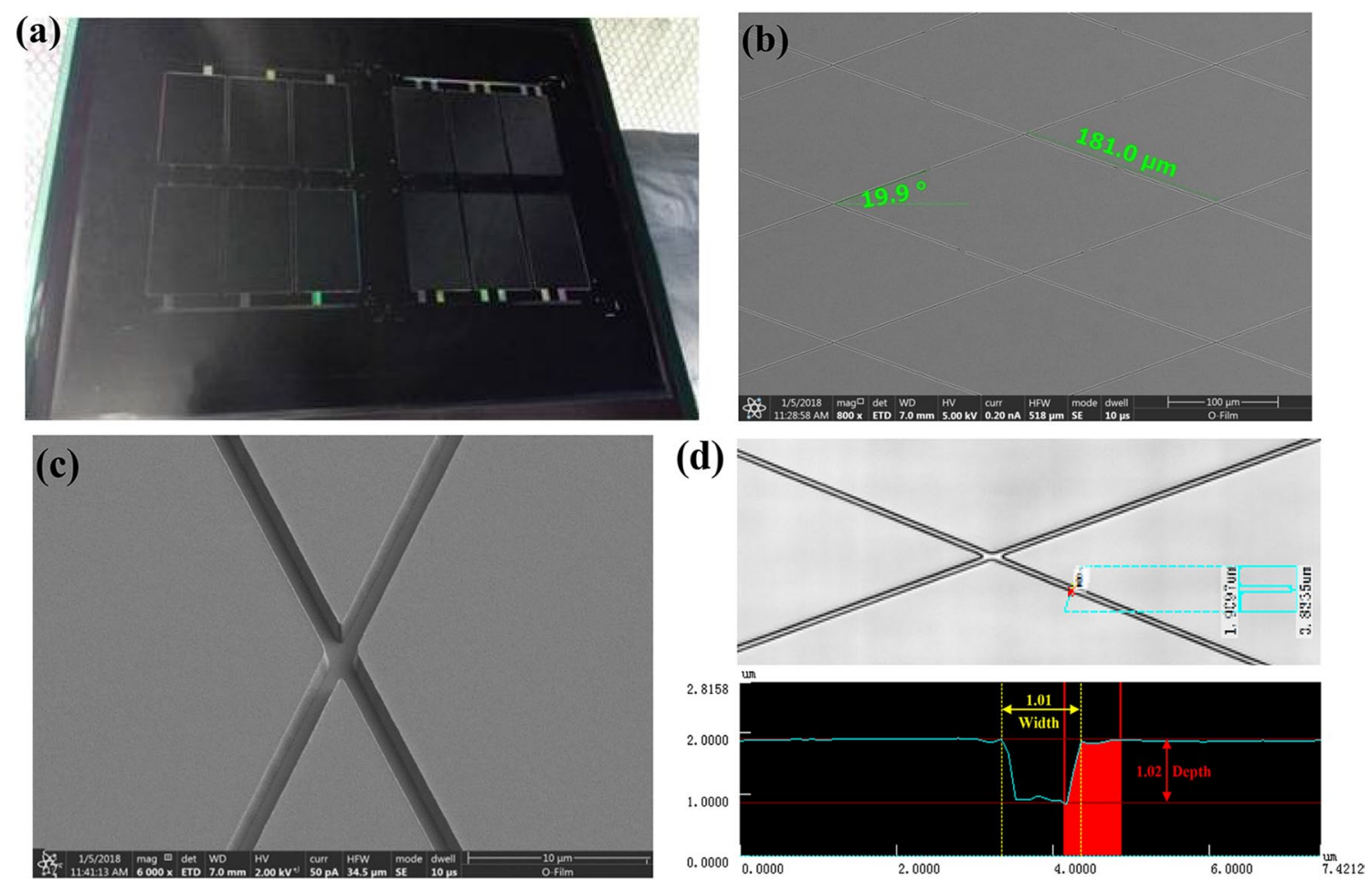

Fig. 3 The images of the micro-grooves on the UV glue imprinted on the PET film in this work: a The macrograph of the pattern on the UV glue; $\mathbf{b}$ The grid size and angle observed with SEM; c The

bevel view of a groove on the UV glue observed with SEM; $\mathbf{d}$ The illustration of measuring the width and depth of the groove on the UV glue observed with SLM

\subsection{Characterization of the ultrafine Ag grid film}

\subsubsection{Morphological characterization}

To make the ultrafine Ag grid film have good electrical conductivity, and effectively reduce the residual of $\mathrm{Ag}$ paste on the non-grooves region during the process of filling grooves with Ag paste, the Ag particle size of the Ag paste in this work mainly distributed in the ranges of 200-300 nm (27.86\%) and 300-400 nm (29.10\%) shown in Fig. 4 was selected, it also can be seen from Fig. 5 that the micro-shapes of the used Ag particles were irregular, the size of the Ag particles was measured by the Mastersizer 3000 Particle Size Analyzer from Malvern Panalytical. Once the Ag paste was drawn into the groove by a doctor blade under a certain pressure and inclination angle, which was filled into the micro-grooves and baked at $130^{\circ} \mathrm{C}$ for $1 \mathrm{~h}$, the ultrafine Ag grid film was generated. The micrograph of the ultrafine Ag grid is shown in Fig. 6a, and it can be seen that the Ag particles were filled fully in the micro-grooves and had good contact with the micro-grooves. The micrograph of the sintered structure of Ag grid is shown in Fig. 6b, the width and depth of a micro-groove is $3.0 \mu \mathrm{m}$, respectively. The measurement of the width and depth of the sintered

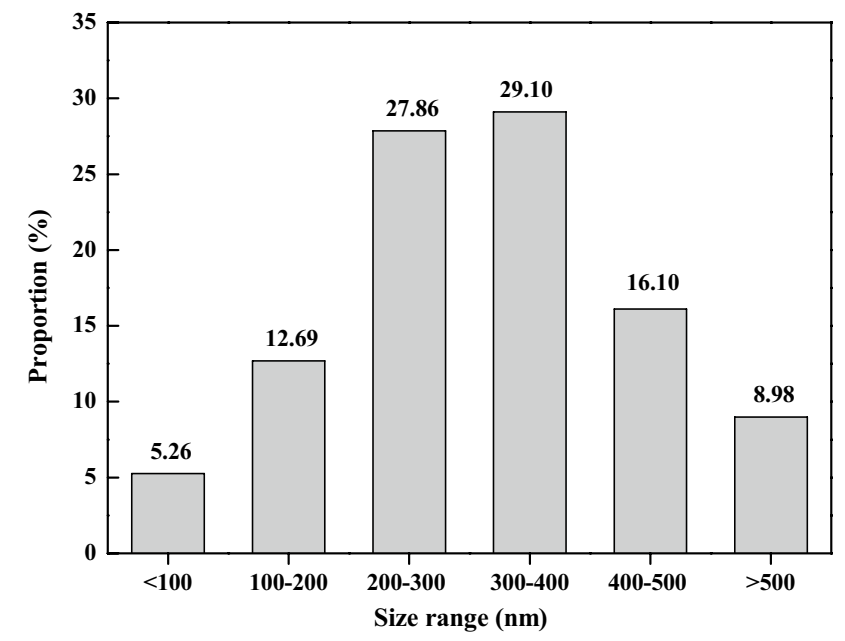

Fig. 4 The column chart of the size distribution of $\mathrm{Ag}$ particles filled into the micro-grooves

structure of Ag grid is $2.841 \mu \mathrm{m}$ and $2.023 \mu \mathrm{m}$, respectively. The percentage of the area is effectively covered with $\mathrm{Ag}$ is calculated by $\mathrm{P}=$ (the width of the sintered structure of Ag grid* the depth of the sintered structure of Ag grid)/(the width of a micro-groove* the depth of a micro-groove), and the percentage is $63.86 \%$. 


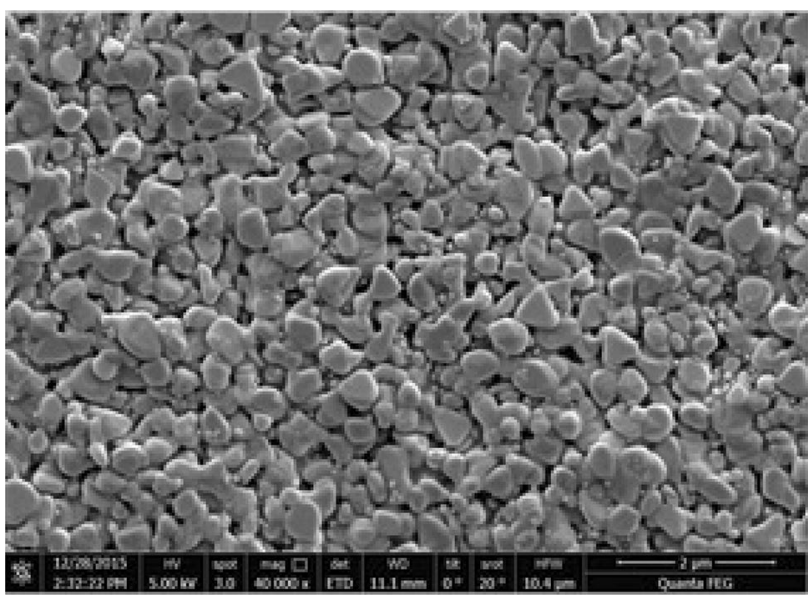

Fig. 5 The morphologies of the used Ag particles observed with SEM

\subsubsection{Optical performance}

The optical properties of PET film and PET $+\mathrm{Ag}$ grid film in the visible wavelengths (380-780 $\mathrm{nm}$ ) are shown in Table 1. It can be seen that the transmittance of PET $+\mathrm{Ag}$ grid film is $90.00 \%$, which is very close to that of PET film (90.21\%). Moreover, it is well known that the values of " $L$ ", "a", "b" reflect the degrees of materials between brightness and darkness, red and green, yellow and blue, respectively. The values of "L", "a", "b" of PET film are 96.13, 0.17 and 0.50, respectively. And those values of $\mathrm{PET}+\mathrm{Ag}$ grid film are $96.09,0.00$ and 0.66 , respectively. This shows that both the PET film and PET + Ag grid film in this work have excellent chromaticity for the application to flexible touch senor. Furthermore, after the filling of Ag paste, the optical reflectance $(\mathrm{R})$ was effectively reduced from 11.43 to $10.34 \%$ in the visible wavelengths, which is an advantage enhancement to the application of the ultrafine Ag grid film to the flexible touch sensor. In addition, based on Fig. 7, it can be concluded that the optical transmittance $(T)$ and reflectance (R) of PET + Ag grid film fluctuate less than those of PET film in visible wavelengths. Although, the haze (1.49\%) of PET + Ag grid film is higher than that (0.34\%) of PET film, which is still an excellent value of haze compared to the haze range of 2-6.1\% reported by Hyunjin et al. [23].

\subsubsection{Mechanical and electrical properties}

For the flexible application, the mechanical stability of the transparent conductive electrode is very important. The mechanical stability of the fabricated ultrafine Ag grid film was detected by cyclic bending test and adhesion cross-cut test. A homemade device for the cyclic bending test is shown in Fig. 8a. The compressive strain was applied for thermomechanical bending, the bending direction was relative to the line pattern, the bending axis passed through the center of the channel and was perpendicular to the channel direction. The device includes two parallel metal plates, one plate is fixed, and the other plate can move back and forth. The bending of the PET + Ag grid film is achieved by fixing one end of the film on the fixed plate of the device, and fixing the other end of the film on the moved plate of the device, which moves at a proper frequency (f). The bending

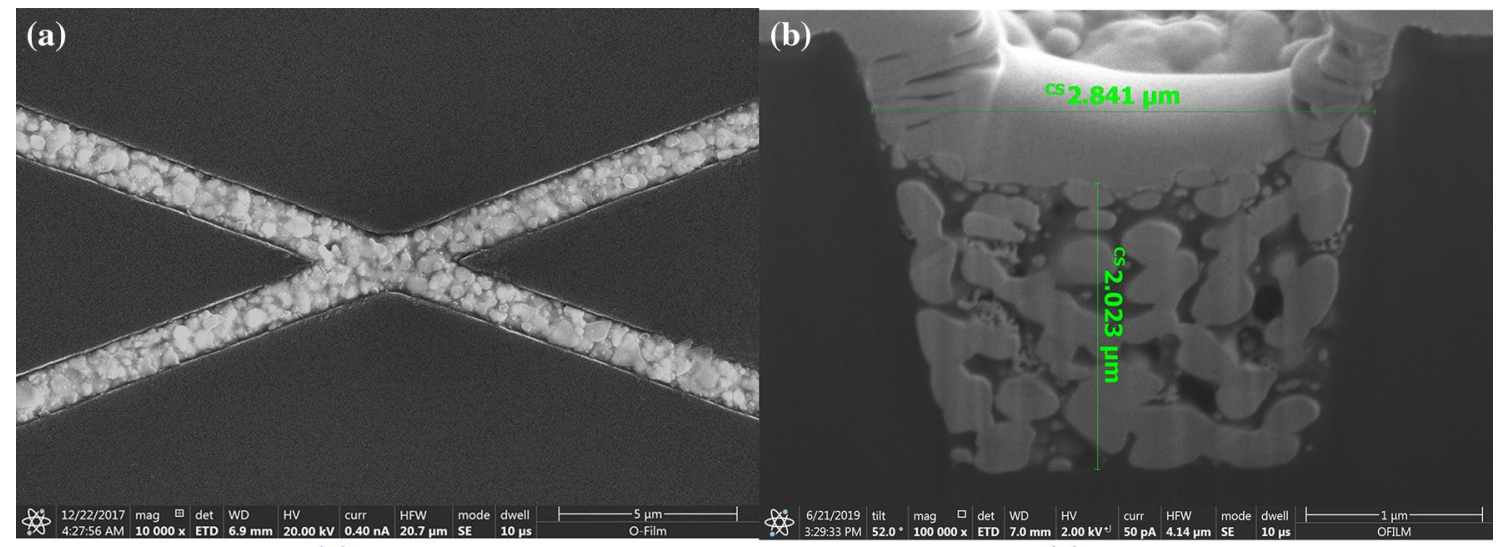

Fig. 6 The micrograph of the ultrafine Ag grid on the UV glue (a) and the sintered structure of Ag grid (b) observed with SEM

Table 1 The optical properties of PET film and PET + Ag grid film

\begin{tabular}{lllllll}
\hline Material & $\mathrm{T}(\%)$ & $\mathrm{L}$ & $\mathrm{a}$ & $\mathrm{b}$ & $\mathrm{R}(\%)$ & $\mathrm{H}(\%)$ \\
\hline PET film & 90.21 & 96.13 & 0.17 & 0.50 & 11.43 & 0.34 \\
PET + Ag grid film & 90.00 & 96.09 & 0.00 & 0.66 & 10.34 & 1.49 \\
\hline
\end{tabular}



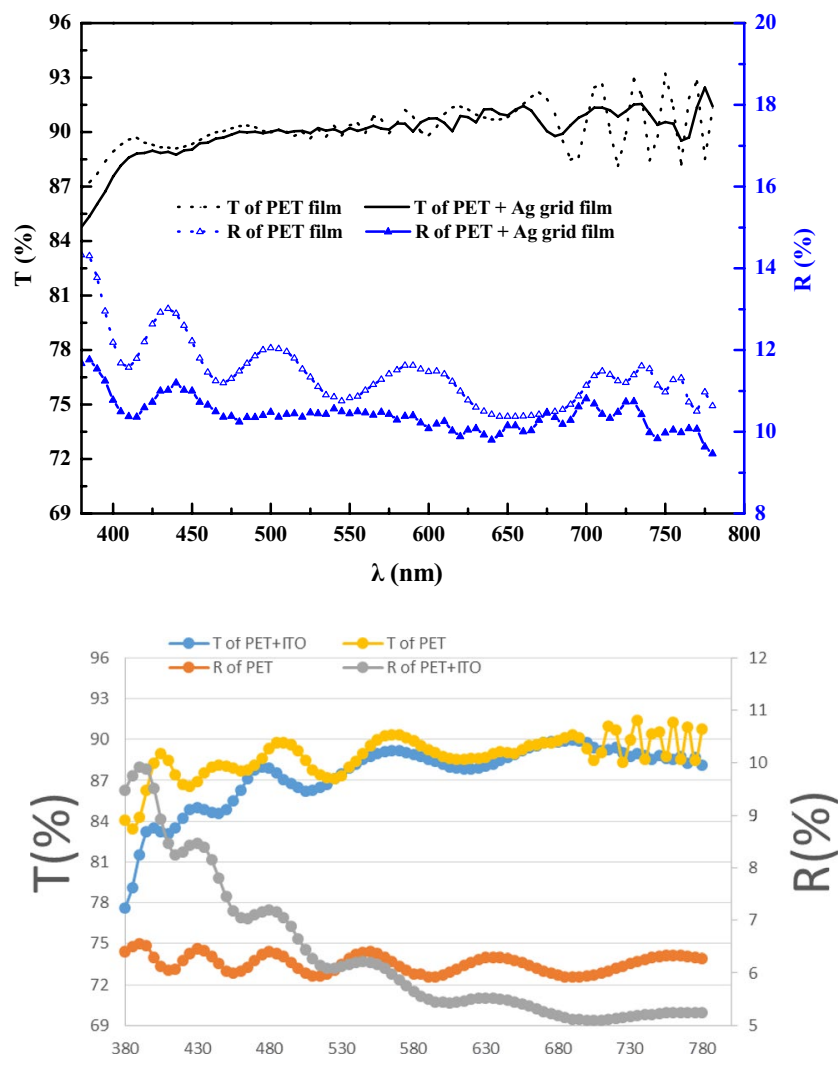

Fig. 7 The plots of optical transmittance and reflectance of PET film, PET + Ag grid film and PET + ITO with wavelength

frequency is $1 \mathrm{~Hz}$, and the resistance was recorded during the bending test. The radius of curvature $(r)$ is $2 \mathrm{~mm}$ at the maximum bending deformation, the distance between the two plates of the device is minimal at the moment. The photographs of the minimum and maximum bending deformations are shown in Fig. 8b, c, respectively. The average sheet resistance is $5.4 \Omega / \square$, which is much lower than most of the reported sheet resistances of transparent conductors used in flexible applications. The plots of the sheet resistance (R $\square$ ) and the corresponding variation ratio of the sheet resistance $\left(\Delta \mathrm{R} \square / \mathrm{R} \square_{, 0}\right)$ with bending times are shown in the Fig. 9. It can be seen from Fig. 9, that the sheet resistance is basically unchanged throughout 1000 bending cycles, the sheet resistance gradually increases throughout 2000 bending cycles, and the changed value of the sheet resistance was within $3 \%$ of the primary sheet resistance (from 5.33 to $5.49 \Omega / \square$ ) throughout 8000 bending cycles, the increased resistance may be the result from the contact resistance between Ag particles. Moreover, compared with the reported data in the literatures, the 8000 bending cycles is $60 \%$ higher than the 5000 bending cycles reported by Hong et al.[22], and the radius of curvature $(2 \mathrm{~mm})$ at the maximum bending deformation is only $2 / 15$ of theirs $(15 \mathrm{~mm})$. 3D microscope shows that the surface roughness of the conductive films is $0.12 \mu \mathrm{m}$, which is a low roughness level, indicating that the conductive film surface is very smooth.

In order to further study the adhesion of the ultrafine Ag grid on PET film, the adhesion cross-cut test was conducted on the PET + Ag grid film. The images of PET $+\mathrm{Ag}$ grid film after the adhesion cross-cut test ( $3 \mathrm{M}$ tape, Scotch) are shown in the Fig. 10. Figure $10 a$, b respect the macrograph and micrograph of PET + Ag grid film after the adhesion cross-cut test, respectively. Based on the images in the Fig. 10, it can be concluded that the change in the morphology of the ultrafine Ag grid film is almost negligible after the adhesion cross-cut test, which not only shows the excellent adhesion between the ultrafine Ag grid and PET film, but also shows the excellent adhesion between the Ag particles making up the ultrafine Ag grid.
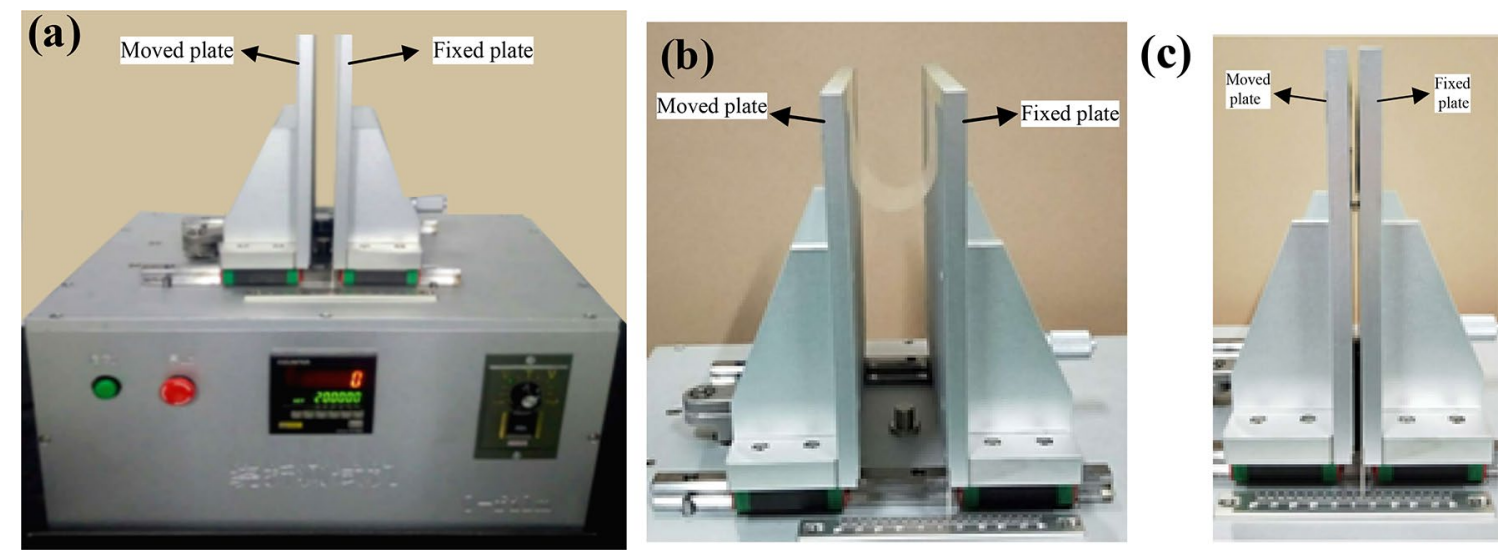

Fig. 8 The images of bending test: a The picture of the homemade device for the cyclic bending test; $\mathbf{b}$ The photograph of the minimum bending deformation of the PET $+\mathrm{Ag}$ grid film; $\mathbf{c}$ The photograph of the maximum bending deformation of the PET $+\mathrm{Ag}$ grid film 


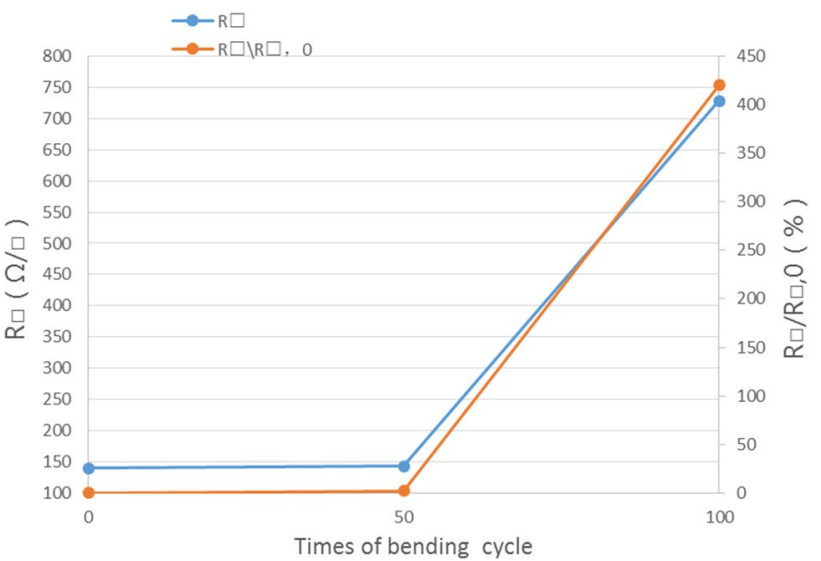

Fig. 9 Plots of sheet resistance and its variation ratio with times of bending cycle in this work

\subsubsection{Functional test}

Based on the above studies, the fabricated ultrafine Ag grid transparent conductive film shows excellent optoelectronic and mechanical performances. Furthermore, the function of the flexible touch sensor applying this film was studied. The functional test was performed on the flexible touch sensor by connecting it with the functional test fixture through a flexible printed circuit board (FPCB). The control chip (ZT7538) in the test fixture was provided by Zinitix Co., Ltd (Korea). The performance of the flexible touch sensor was presented by writing the letters "O-film", Wavy lines and straight lines on it, as shown in the Fig. 11. It can be seen that the letters and lines are well displayed on the display, and the widths of the lines on display could be changed smoothly when the writing force of the pen changed. Thus, it can be concluded that the

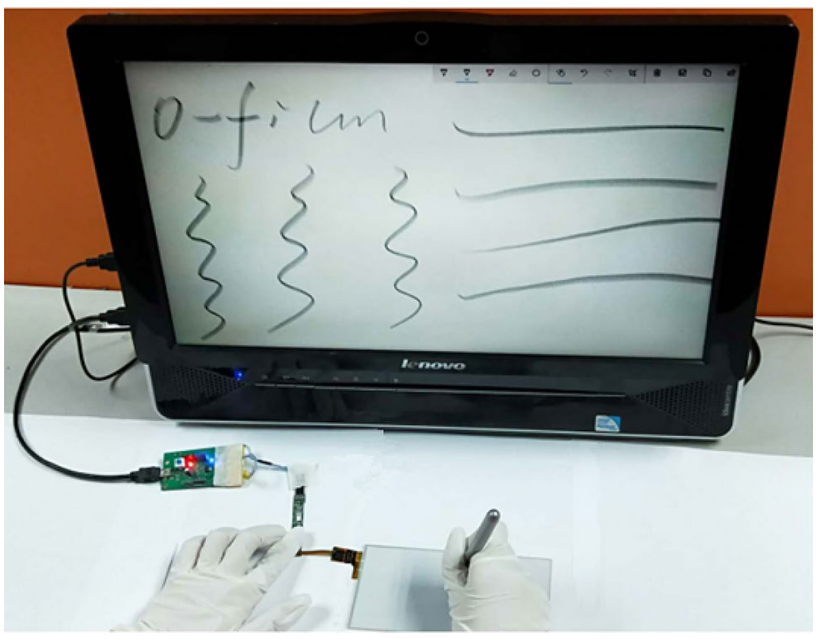

Fig. 11 The flexible touch sensor under functional test

fabricated flexible touch sensor worked successfully, and the fabricated ultrafine Ag grid film is a promising transparent conductive electrode that can substitute for the conventional ITO electrode applied in the large-area, high-throughput and low-cost production of flexible touch sensor.

\section{Conclusions}

In summary, we have demonstrated the fabrication and performance of an ultrafine Ag grid film applied to flexible touch sensor as the transparent conductive electrode. The ultrafine Ag grid film was fabricated based on the laser direct writing, electroforming and nano-imprint lithography, and the maximum size of the ultrafine Ag grid transparent conductor is $(\mathrm{L})$

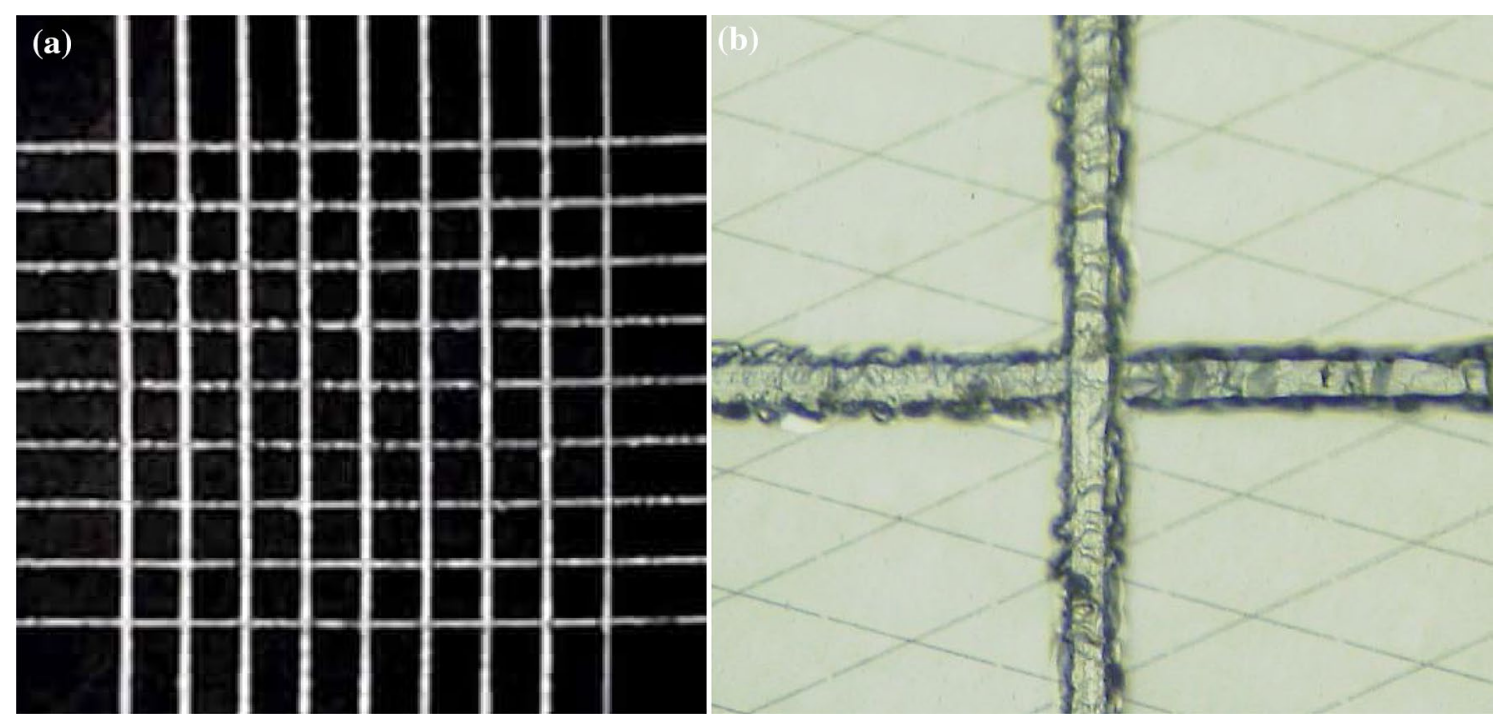

Fig. 10 The images of the grid pattern on the touch sensor module after the adhesive tape test: $\mathbf{a}$ The macrograph; $\mathbf{b}$ The micrograph

\section{SN Applied Sciences


$640 \mathrm{~mm} \times(\mathrm{W}) 520 \mathrm{~mm}$. The fabricated ultrafine Ag grid transparent conductive film shows excellent optoelectronic and mechanical properties, such as line width $1.01 \mu \mathrm{m}$, transmittance $90.00 \%$, haze $1.49 \%$, sheet resistance $5.4 \Omega / \square$, the variation ratio of the sheet resistance within $3 \%$ after 8000 bending cycles and the almost negligible morphology change after the adhesion cross-cut test. Moreover, the flexible touch sensor applying this film was fabricated and worked successfully, as well as could identify the writing force. Thus, the fabricated ultrafine Ag grid film is a promising transparent conductive electrode that can substitute for the conventional ITO electrode applied in the large-area, high-throughput and low-cost production of flexible touch sensor.

Acknowledgements The financial supports from the research and development projects of O-Film Technology Co., Ltd. are gratefully acknowledged.

\section{Compliance with ethical standards}

Conflict of interest The authors declare that they have no conflict of interest.

Open Access This article is licensed under a Creative Commons Attribution 4.0 International License, which permits use, sharing, adaptation, distribution and reproduction in any medium or format, as long as you give appropriate credit to the original author(s) and the source, provide a link to the Creative Commons licence, and indicate if changes were made. The images or other third party material in this article are included in the article's Creative Commons licence, unless indicated otherwise in a credit line to the material. If material is not included in the article's Creative Commons licence and your intended use is not permitted by statutory regulation or exceeds the permitted use, you will need to obtain permission directly from the copyright holder. To view a copy of this licence, visit http://creativecommons .org/licenses/by/4.0/.

\section{References}

1. Chung SI, Pan KK, Kwon YW, Ha TG, Hur JW, Lee KM (2016) Fabrication of metallic nanomesh structures using phase shift lithography and its application to touch screen panels. J Mater Process Technol 238:431-436

2. Yang L, Zhang T, Zhou H, Price SC, Wiley BJ, You W (2011) Solution-processed flexible polymer solar cells with silver nanowire electrodes. ACS Appl Mater Interfaces 3:4075-4084

3. Blake $P$, Brimicombe PD, Nair RR, Booth TJ, Jiang D, Schedin F, Ponomarenko LA, Morozov SV, Gleeson HF, Hill EW (2008) Graphene-based liquid crystal device. Nano Lett 8:1704-1708

4. Wu J, Agrawal $M$, Becerril HA, Bao Z, Liu Z, Chen Y, Peumans $P(2010)$ Organic light-emitting diodes on solution-processed graphene transparent electrodes. ACS Nano 4:43-48

5. Jeon $Y$, Han BJ, Jung $S$, Go H, Lee I, Lee C, Joo YK, Park K (2015) Highly flexible touch screen panel fabricated with silver nanowire crossing electrodes and transparent bridges. J Opt Soc Korea 19:508-513

6. Kim DJ, Shin HI, Ko EH, Kim KH, Kim TW, Kim HK (2016) Roll-toroll slot-die coating of $400 \mathrm{~mm}$ wide, flexible, transparent $\mathrm{Ag}$ nanowire films for flexible touch screen panels. Sci Rep 6:34322
7. Hecht DS, Hu L, Irvin G (2011) Emerging transparent electrodes based on thin films of carbon nanotubes, graphene, and metallic nanostructures. Adv Mater 23:1482-1513

8. Kwon N, Kim K, Sung S, Yi I, Chung I (2013) Highly conductive and transparent Ag honeycomb mesh fabricated using a monolayer of polystyrene spheres. Nanotechnology 24:235205

9. Zhou L, Xiang HY, Shen S, Li YQ, Chen JD, Xie HJ, Goldthorpe IA, Chen LS, Lee ST, Tang JX (2014) High-performance flexible organic light-emitting diodes using embedded silver network transparent electrodes. ACS Nano 8:12796-12805

10. Li YW, Meng L, Yang Y, Xu GY, Hong Z, Chen Q, You JB, Li G, Li YF (2016) High-efficiency robust perovskite solar cells on ultrathin flexible substrates. Nat Commun 7:10214. https://doi. org/10.1038/ncomms10214

11. Yi PY, Zhang CP, Peng LF, Lai XM (2017) Flexible silver-mesh electrodes with moth-eye nanostructures for transmittance enhancement by double-sided roll-to-roll nanoimprint lithography. RSC Adv 7:48835-48840

12. Yu Z, Niu X, Liu Z, Pei Q (2011) Intrinsically stretchable polymer light-emitting devices using carbon nanotube-polymer composite electrodes. Adv Mater 23:3989-3994

13. Bae S, Kim H, Lee Y, Xu X, Park JS, Zheng Y, Balakrishnan J, Lei T, Kim HR, Song YI (2010) Roll-to-roll production of 30-inch graphene films for transparent electrodes. Nat Nanotechnol 5:574-578

14. Tung VC, Allen MJ, Yang Y, Kaner RB (2009) High-throughput solution processing of large-scale graphene. Nat Nanotechnol 4:25-29

15. Kirchmeyer S, Reuter K (2005) Scientific importance, properties and growing applications of poly(3,4-ethylenedioxythiophene). J Mater Chem 15:2077-2088

16. Xiong WW, Liu HL, Chen YZ, Zheng ML, Zhao YY, Kong XB, Wang $Y$, Zhang XQ, Kong XY, Wang PF, Jiang L (2016) Highly conductive, air-stable silver nanowire@longel composite films toward flexible transparent electrodes. Adv Mater 28:7167. https://doi. org/10.1002/adma.201600358

17. Chang L, Zhang $X Q$, Ding $Y$, Liu HL, Liu MZ, Jiang L (2018) lonogel/Copper grid composites for high-performance, ultra-stable flexible transparent electrodes. ACS Appl Mater Interfaces 10:29010-29018

18. Gaynor W, Burkhard GF, Mcgehee MD, Peumans P (2011) Smooth nanowire/polymer composite transparent electrodes. Adv Mater 23:2905-2910

19. Hong S, Yeo J, Lee J, Lee H, Lee P, Lee SS, Ko SH (2015) Selective Laser Direct Patterning of Silver Nanowire Percolation Network Transparent Conductor for Capacitive Touch Panel. J Nanosci Nanotechnol 15:2317-2323

20. Madaria AR, Kumar A, Zhou C (2011) Large scale, highly conductive and patterned transparent films of silver nanowires on arbitrary substrates and their application in touch screens. Nanotechnology 22:245201

21. Kumar A, Zhou C (2010) The race to replace tin-doped indium oxide: which material will win? ACS Nano 4:11-14

22. Hong S, Yeo J, Kim G, Kim D, Lee H, Kwon J, Lee H, Lee P, Ko SH (2013) Nonvacuum, maskless fabrication of a flexible metal grid transparent conductor by low-temperature selective laser sintering of nanoparticle ink. ACS Nano 7:5024-5031

23. Hyunjin M, Phillip W, Jinhwan L, Seung HK (2016) Low-haze, annealing-free, very long Ag nanowire synthesis and its application in a flexible transparent touch panel. Nanotechnology 27:295201

Publisher's Note Springer Nature remains neutral with regard to jurisdictional claims in published maps and institutional affiliations. 